\title{
Optimal Averaging Procedures in Almost Sure Central Limit Theory
}

\author{
Siegfried Hörmann ${ }^{1}$
}

\begin{abstract}
Let $X_{1}, X_{2}, \ldots$ be i.i.d. random variables with $E X_{1}=0, E X_{1}^{2}=1, S_{n}=$ $X_{1}+\ldots+X_{n}$ and let $\left(d_{k}\right)$ be a positive numerical sequence. We investigate the a.s. convergence of the averages

$$
\frac{1}{D_{N}} \sum_{k=1}^{N} d_{k} I\left\{S_{k} / \sqrt{k} \leq x\right\},
$$

where $D_{N}=\sum_{k=1}^{N} d_{k}$. In the case of $d_{k}=1 / k$ we have logarithmic means and by the almost sure central limit theorem the above averages converge a.s. to $\Phi(x)$, the standard normal distribution function. It is also known that the analogous convergence relation fails for $d_{k}=1$ (ordinary averages). In this paper we give a fairly complete solution of the problem for which weight sequences the above convergence relation and its refinements hold.
\end{abstract}

\section{Introduction}

\subsection{The almost sure central limit theorem}

One of the most frequently investigated topics in classical probability theory is the fluctuation of the partial sums $S_{k}$ of i.i.d. random variables $X_{1}, X_{2}, \ldots$ In case of $E X_{1}=0$ and $E X_{1}^{2}=1$ the central limit theorem (CLT) yields that

$$
P\left\{k^{-1 / 2} S_{k} \leq x\right\} \longrightarrow \Phi(x) \quad(k \rightarrow \infty) .
$$

Hence one might expect that the average time the path of the process $\left(k^{-1 / 2} S_{k}\right)_{k \geq 1}$ spends below $x \in \mathbb{R}$ is asymptotically $\Phi(x)$, i.e.

$$
\frac{1}{N} \sum_{1 \leq k \leq N} I\left\{k^{-1 / 2} S_{k} \leq x\right\} \rightarrow \Phi(x) \quad \text { a.s. } \quad(N \rightarrow \infty) .
$$

Of course, the random variables $I\left\{k^{-1 / 2} S_{k} \leq x\right\}$ are not independent and have also no other structural property which would allow the application of the law of large numbers

\footnotetext{
${ }^{1}$ Department of Statistics, Steyrergasse 17/IV, University of Technology Graz, Austria; shoermann@tugraz.at
} 
(LLN). And indeed (1.1) is not true! The easiest way to see this is to observe that by Lévy's arc-sine law we have

$$
\frac{1}{N} \sum_{1 \leq k \leq N} I\left\{S_{k} \leq 0\right\} \stackrel{\mathcal{L}}{\longrightarrow} T
$$

where $T$ is the arc-sine distribution with density

$$
a(x)=\frac{1}{\pi} \frac{1}{\sqrt{x(1-x)}} \quad 0<x<1 .
$$

Note that $a(x)$ takes its minimum at $x=1 / 2$ and becomes infinite at 0 and 1 . Thus for fixed large $N$ the least likely value of the fraction of time the path $\left(k^{-1 / 2} S_{k}\right)_{k=1}^{N}$ spends on the positive half axis is $1 / 2$, and it is much more likely that the path remains most of the time either below or above the $x$-axis rather than to level off to an equilibrium.

A possible way to overcome the strong dependence of the random variables $I\left\{S_{k} \leq\right.$ $0\}$ has been shown by Erdős and Hunt (1953). They proved that if $X_{1}$ has a continuous and symmetric distribution function then

$$
\lim _{N \rightarrow \infty} \frac{1}{\log N} \sum_{k=1}^{N} \frac{1}{k} I\left\{S_{k} \leq 0\right\}=1 / 2 \text { a.s. }
$$

The difference between (1.2) and (1.3) lies in the summation method. In the latter we used logarithmic means instead of the common Cesàro means. This result is a special case of the so called almost sure central limit theorem (ASCLT) proved first by Brosamler (1988) and Schatte (1988). (Actually, a very similar result has been already stated by Lévy in 1937 (cf. Lévy, 2003), but without a proof). In order to formulate this result, let $(\Omega, \mathcal{F}, P)$ be the probability space on which the sequence $\left(X_{n}\right)$ is defined.

Theorem A Let $X_{1}, X_{2}, \ldots$ be a sequence of i.i.d. random variables with $E X_{1}=0$, $E X_{1}^{2}=1$ and define $S_{k}=X_{1}+\cdots+X_{k}$. Then there is a set $N \in \mathcal{F}$ with $P(N)=0$ such that for any Borel set $A \subset \mathbb{R}$ with $\lambda(\partial A)=0$

$$
\frac{1}{\log N} \sum_{k=1}^{N} \frac{1}{k} I\left\{k^{-1 / 2} S_{k} \in A\right\} \rightarrow \frac{1}{\sqrt{2 \pi}} \int_{A} e^{-t^{2} / 2} d t \quad \text { on } \Omega \backslash N .
$$

Brosamler and Schatte proved Theorem A assuming $E\left|X_{1}\right|^{2+\delta}<\infty$ for some $\delta>0$; for a proof assuming only finite second moments, see Lacey and Philipp (1990). In the past 15 years, a wide literature dealt with various extensions of the ASCLT; for a survey we refer to Berkes (1998).

The crucial new feature of the a.s. central limit theorem (1.4) is its pathwise character: the limit of the left hand side of (1.4) can be computed for any fixed $\omega \in \Omega$ knowing only the values of $X_{n}, n=1,2, \ldots$ at $\omega$. Hence the ASCLT is often called pathwise central limit theorem.

The following lemma will be the basis for a heuristic proof of Theorem A. 
Lemma 1 Let $(\mathbb{W}(t))_{t \geq 0}$ be a standard Brownian motion process and let $f$ be a continuous function. Then

$$
\frac{1}{\log T} \int_{1}^{T} \frac{1}{t} f\left(t^{-1 / 2} \mathbb{W}(t)\right) d t \rightarrow E f(\mathbb{W}(1)) \quad \text { a.s. }
$$

Proof. By parameter transformation we get

$$
\frac{1}{\log T} \int_{1}^{T} \frac{1}{t} f\left(t^{-1 / 2} \mathbb{W}(t)\right) d t=\frac{1}{\log T} \int_{0}^{\log T} f(Z(t)) d t
$$

where $Z(t)=e^{-t / 2} \mathbb{W}\left(e^{t}\right)$ is (the stationary) Ornstein-Uhlenbeck process. Hence the result follows from the ergodic theorem.

Using Lemma 1, Theorem A can be deduced now by an invariance argument. Assume that $f$ is uniformly continuous. Replacing

$$
t^{-1} f\left(t^{-1 / 2} \mathbb{W}(t)\right) \quad \text { by } \quad k^{-1} f\left(k^{-1 / 2} \mathbb{W}(k)\right) \quad \text { if } t \in[k, k+1)
$$

does not harm the limiting behavior in (1.5). Now assuming slightly more than two moments for $X_{1}$, by an almost sure invariance principle (cf. Csörgố and Révész, 1981: 108) we can redefine $\mathbb{W}$ and $X_{1}, X_{2}, \ldots$ jointly on a new probability space in such a way that

$$
k^{-1 / 2} \mathbb{W}(k)-k^{-1 / 2} S_{k}=o(1) \quad \text { a.s. }
$$

Since $f$ is uniformly continuous, it follows that

$$
f\left(k^{-1 / 2} \mathbb{W}(k)\right)-f\left(k^{-1 / 2} S_{k}\right) \rightarrow 0 \quad \text { a.s. } \quad(k \rightarrow \infty) .
$$

Finally, it is not hard to see that by an approximation argument the result of Lemma 1 extends to indicator functions $f=I_{A}$ where $A \subset \mathbb{R}$ is a Borel set with $\lambda(\partial A)=0$.

\subsection{Linear summation methods}

The argument above shows why logarithmic summation in the ASCLT is natural from an ergodic point of view. Note that logarithmic summation is stronger than Cesàro summation, i.e. if for a numerical sequence $\left(x_{k}\right)_{k \geq 1}$ we have

$$
\lim _{N \rightarrow \infty} \frac{1}{N} \sum_{k=1}^{N} x_{k}=x \quad \text { (say), }
$$

then

$$
\lim _{N \rightarrow \infty} \frac{1}{\log N} \sum_{k=1}^{N} \frac{1}{k} x_{k}=x .
$$

That the converse is false can be seen by defining

$$
x_{k}=\left\{\begin{array}{lll}
0 & \text { if } \quad k \in\left\{2^{2 i}, \ldots, 2^{2 i+1}-1\right\} \\
1 & \text { if } \quad k \in\left\{2^{2 i+1}, \ldots, 2^{2(i+1)}-1\right\}
\end{array} \quad(i \geq 0) .\right.
$$


In some sense, this sequence $\left(x_{k}\right)$ exhibits a similar behavior as $\left(I\left\{k^{-1 / 2} S_{k} \leq x\right\}\right)$. Long periods of 0 's are followed by even longer periods of 1's and conversely. Let $x=0$. By the CLT the probability that $k^{-1 / 2} S_{k}$ lies outside the interval $(-\epsilon, \epsilon)$ is close to 1 for small $\epsilon>0$. If we assume that $\left|X_{k}\right| \leq 1$ for all $k$, then it will take at least $n>\epsilon \sqrt{k}$ steps until $I\left\{k^{-1 / 2} S_{k} \leq 0\right\}$ will change from 0 to 1 or the other way round. In order to compensate this persistence in the same state we have to weigh down the random variables $I\left\{k^{-1 / 2} S_{k} \leq x\right\}$. Thus, the explanation that logarithmic means are a proper tool in this theory might also result from the point of view of summability theory. By this argument, one should also expect to get stronger results if we use larger weights.

The elegance of the above heuristic argument for Theorem A raises the question if there exist other, substantially different averaging methods which also work in the ASCLT. Let us note first that log averaging is not unique: it was observed by Peligrad and Révész (1991) that

$$
\frac{\alpha+1}{(\log N)^{\alpha+1}} \sum_{k=1}^{N} \frac{(\log k)^{\alpha}}{k} I\left\{k^{-1 / 2} S_{k} \in A\right\} \rightarrow \Phi(A) \quad \text { a.s. }
$$

for any $\alpha>-1$. While the averaging in (1.6) is rather similar to log averages, Berkes and Csáki (2001) showed that

$$
\frac{1}{D_{N}} \sum_{k=1}^{N} d_{k} I\left\{k^{-1 / 2} S_{k} \in A\right\} \rightarrow \Phi(A) \quad \text { a.s. }
$$

for

$$
d_{k}=\exp \left((\log k)^{\alpha}\right) k^{-1}, \quad D_{k}=\sum_{j=1}^{k} d_{j} \quad(0 \leq \alpha<1 / 2) .
$$

(Also, (1.7) holds for all smaller weight sequences $\left(d_{k}\right)$ as well). To understand the connection between these results and the standard ASCLT (1.4), we shall review some facts on linear summation methods.

Let $\mathbf{D}=\left(D_{N}\right)$ be a positive non-decreasing sequence with $\lim _{N \rightarrow \infty} D_{N}=\infty$ and set $d_{k}=D_{k}-D_{k-1}$. We say that $\left(x_{k}\right)_{k \geq 1}$ is $\mathbf{D}$-summable to $x$ if

$$
\lim _{N \rightarrow \infty} \frac{1}{D_{N}} \sum_{k=1}^{N} d_{k} x_{k}=x
$$

By a result of Hardy (see e.g. Chandrasekharan and Minakshisundaram, 1952: 35), if D and $\mathbf{D}^{*}$ are summation procedures with $D_{N}^{*}=O\left(D_{N}\right)$, then under minor technical assumptions, the summation $\mathbf{D}^{*}$ is stronger than $\mathbf{D}$, i.e. if a sequence $\left(x_{n}\right)$ is $\mathbf{D}$-summable to $x$, then it is also $\mathbf{D}^{*}$-summable to $x$. Also, if $\left(d_{k}\right)$ grows exponentially or faster, then (1.9) is equivalent to convergence of the sequence $\left(x_{k}\right)$, and hence this is the weakest summation method. By a result of Zygmund (see also Chandrasekharan and Minakshisundaram, 1952: 35) if $D_{N}^{\alpha} \leq D_{N}^{*} \leq D_{N}^{\beta}\left(N \geq N_{0}\right)$ for some $\alpha>0, \beta>0$, then $\mathbf{D}$ and $\mathbf{D}^{*}$ are equivalent, and if $D_{N}^{*}=O\left(D_{N}^{\varepsilon}\right)$ for any $\varepsilon>0$, then $\mathbf{D}^{*}$ is strictly stronger than $\mathbf{D}$. These results show that the larger the norming sequence $D_{N}$ in (1.9) is, the stronger the relation (1.9) becomes. In view of Zygmund's theorem, (1.6) is actually equivalent to Theorem A. 
Note also that the larger weights

$$
d_{k}=k^{-\alpha}, \quad D_{k}=\sum_{j=1}^{k} d_{j} \quad(\alpha<1)
$$

define a summation equivalent to ordinary averaging, and hence do not work in the ASCLT. On the other hand, the result of Berkes and Csáki provides summation methods in the ASCLT which are strictly weaker than logarithmic summation and thus yield a sharpening of the ordinary ASCLT. The summation procedures defined by (1.8) lie strictly between $\log$ and Cesàro averaging and are pairwise nonequivalent for different $\alpha$ 's. Note that for $\alpha=0$, resp. $\alpha=1$ (1.8) reduces to logarithmic, resp. ordinary averaging and thus the result of Berkes and Csáki shows that the ASCLT remains valid at least until 'halfway' from logarithmic to ordinary averaging. In view of Hardy's result the 'true' form of the ASCLT is given by relation (1.7) where the $D_{N}$ is maximal, i.e. $D_{N}$ is the weakest summation method. This maximal $D_{N}$ is unknown. In the following section we will give substantial improvements of the result in Berkes and Csáki: we will show that the ASCLT (1.7) holds under a fairly general growth condition on $\left(d_{k}\right)$, similar to the condition in Kolmogorov's LIL. In particular, our results will imply that the sequence (1.8) obeys the ASCLT for all $0 \leq \alpha<1$, and thus the critical summation procedure in a.s. central limit theory lies much closer, in some sense, to ordinary averaging. We will then determine fairly sharply the critical summation method $\mathbf{D}$.

\section{Results}

Our first theorem is formulated in a more general setting. We will assume that $X_{n}$ are independent, but we drop the assumption that they are identically distributed or have finite second moments.

Theorem 1 Let $X_{1}, X_{2}, \ldots$ be independent random variables with partial sums $S_{n}$ and assume that for some numerical sequences $a_{n}>0$ and $b_{n}$ we have

$$
\frac{S_{n}}{a_{n}}-b_{n} \stackrel{\mathcal{L}}{\longrightarrow} H
$$

with some (possibly degenerate) distribution function $H$. Assume that

$$
E\left|\frac{S_{n}}{a_{n}}-b_{n}\right|^{p}=O(1) \quad(n \rightarrow \infty),
$$

and

$$
a_{k} / a_{l} \leq C(k / l)^{\beta} \quad(1 \leq k \leq l)
$$

for some positive constants $p, \beta, C$. Assume finally that $k d_{k} \gg 1$ and $d_{k} k^{\alpha}$ is eventually non-increasing for some $0<\alpha<1$ and that for some $\rho>0$

$$
d_{k}=O\left(\frac{D_{k}}{k\left(\log D_{k}\right)^{\rho}}\right)
$$


Then if $f$ is a bounded Lipschitz 1 function or an indicator function of a Borel set $A$ with $\lambda(\partial A)=0$, we have

$$
\lim _{N \rightarrow \infty} D_{N}^{-1} \sum_{k=1}^{N} d_{k} f\left(\frac{S_{k}}{a_{k}}-b_{k}\right)=\int_{-\infty}^{\infty} f(x) d H(x) \text { a.s. }
$$

Conditions (2.1)-(2.3) are satisfied e.g. if $X_{k}$ are i.i.d. with finite second moments or if $X_{k}$ are i.i.d. random variables belonging to the domain of attraction of a stable distribution $H$. In this case $\left(a_{k}\right)$ is regularly varying with exponent $1 / \alpha$ for some $0<\alpha \leq 2$ and the validity of relation (2.3) follows easily from the representation theorem for regularly varying functions. Conditions (2.1)-(2.2) are also satisfied if $X_{k}$ are independent r.v.'s with $E X_{k}=0, E X_{k}^{2}<\infty$ satisfying the Lindeberg condition

$$
\lim _{n \rightarrow \infty} s_{n}^{-2} \sum_{k=1}^{n} E X_{k}^{2} I\left(\left|X_{k}\right| \geq \varepsilon s_{n}\right)=0 \quad \text { for all } \varepsilon>0,
$$

where $s_{n}^{2}=\sum_{k=1}^{n} E X_{k}^{2}$. In this case we can choose $a_{k}=s_{k}, b_{k}=0$ and the additional assumption (2.3) is essential: as an example in Berkes and Dehling (1993) shows, without (2.3) the theorem fails even if $d_{k}=1 / k$.

Proposition 1 Assume that relation (2.4) of Theorem 1 is satisfied for some sequence $\left(D_{N}\right)$. Then it is also satisfied for any other sequence $D_{N}^{*}=\psi\left(D_{N}\right)$ provided $\psi$ : $\mathbb{R}^{+} \rightarrow \mathbb{R}^{+}$is differentiable, $\psi^{\prime}(x)=O(\psi(x) / x)$ for $x \rightarrow \infty$ and $\log \psi^{\prime}(x)$ is uniformly continuous on $(A, \infty)$ for some $A>0$. In particular, (2.4) is satisfied for $D_{N}=$ $\exp \left((\log N)^{\alpha}\right), 0 \leq \alpha<1$.

We assumed here implicitly that $\psi(x) \rightarrow \infty$ since otherwise $D_{N}^{*}$ defines of course no summation method. Typical examples for functions $\psi$ permitted in the Proposition are $\psi(x)=x^{\alpha}$ or $\psi(x)=(\log x)^{\alpha}$ when $\alpha>0$.

As we have mentioned above, replacing the weights $d_{k}$ by smaller ones (subject to regularity conditions) leads to a stronger averaging procedure and thus it preserves the validity of the ASCLT (1.7). The second statement of Proposition 1 illustrates the type of regularity condition required in this context. Proposition 1 can be deduced from a theorem of Hirst in summation theory (see Chandrasekharan and Minakshisundaram, 1952: 37-38), but can also be deduced directly from the proof of Theorem 1 . In view of the possibility of replacing the $d_{k}$ with smaller ones, the assumption that $k d_{k} \gg 1$ is no real restriction of generality in Theorem 1. While excluding some irregular weight sequences the assumption $d_{k} k^{\alpha}$ is non-increasing for some $0<\alpha<1$ does also not make any restriction on the order of magnitude of $\left(d_{k}\right)$. Indeed, as we have seen, the sequence $d_{k}=k^{-\alpha}, \alpha>-1$, is already too large to imply the ASCLT.

Until now, on the independent sequence $\left(X_{n}\right)$ we assumed only that its partial sums $S_{n}$, properly centered and normalized, converge weakly. Under the assumption that $X_{n}$ are i.i.d. with finite variance, the sequence $D_{N}=\exp \left((\log N)^{\alpha}\right)$ obtained in Proposition 1 can be made essentially larger, and in fact a nearly optimal summation method can be obtained. Let us note, as we observed above, that the sequence $D_{N}=N^{\alpha}, \alpha>0$, is too large in relation (1.7), and thus it is no restriction on the order of magnitude of $D_{N}$ 
to assume that $D_{N}$ is slowly varying. By the theory of regular variation, $D_{N}$ can then be represented in the form

$$
D_{N}=c_{N} \exp \left(\int_{A}^{N} \epsilon(u) / u d u\right)
$$

where $A>0, c_{N} \rightarrow c \in(0,+\infty), \epsilon(x) \rightarrow 0$ for $x \rightarrow \infty$. Let $\mathcal{W}$ denote the set of sequences $\left(D_{N}\right)$ that can be represented as in (2.6) such that $\epsilon$ is monotone decreasing and obeys additionally

$$
\epsilon(x) / \epsilon\left(x^{2}\right)=O(1) \quad(x \rightarrow \infty) .
$$

Since $\epsilon(x)=(\log x)^{-2}$ is already leading to a bounded $D_{N}$, the last assumption on $\epsilon$ is satisfied in all cases of interest.

Theorem 2 Let $X_{1}, X_{2}, \ldots$ be i.i.d. random variables with $E X_{1}=0, E X_{1}^{2}=1$ and let $S_{n}=X_{1}+\cdots+X_{n}$. Let $\mathbf{D} \in \mathcal{W}$. Assume that

$$
d_{k}=O\left(\frac{D_{k}}{k(\log \log k)^{\alpha}}\right) \quad \text { with } \alpha>3,
$$

and

$$
\left(k d_{k}\right) \text { is eventually non-decreasing. }
$$

Then for any bounded function $f$ which is either Lipschitz 1 or an indicator function of a Borel set $A \subset \mathbb{R}$ with $\lambda(\partial A)=0$, we have

$$
\lim _{N \rightarrow \infty} D_{N}^{-1} \sum_{k=1}^{N} d_{k} f\left(k^{-1 / 2} S_{k}\right)=\int_{-\infty}^{\infty} f(x) d \Phi(x) \quad \text { a.s. }
$$

Theorem 2 yields a strong law of large numbers for the sums $\sum_{k=1}^{N} d_{k} f\left(k^{-1 / 2} S_{k}\right)$. Actually, much more is valid: under conditions similar to Theorem 2 , the weighted sums of $f\left(k^{-1 / 2} S_{k}\right)$ satisfy also the central limit theorem and the law of the iterated logarithm.

Theorem 3 Assume that the conditions of Theorem 2 are satisfied with $\alpha>3$ in (2.7) replaced by $\alpha>1$. Then we have for every non-constant Lipschitz 1 function $f$

$$
\lambda_{N}^{-1 / 2} \sum_{k=1}^{N} d_{k}\left(f\left(\frac{S_{k}}{\sqrt{k}}\right)-E f\left(\frac{S_{k}}{\sqrt{k}}\right)\right) \stackrel{\mathcal{L}}{\longrightarrow} N(0,1)
$$

where

$$
\lambda_{N}:=\operatorname{Var}\left(\sum_{k=1}^{N} d_{k} f\left(\frac{S_{k}}{\sqrt{k}}\right)\right) .
$$

Theorem 4 Assume that the conditions of Theorem 2 are satisfied. Then we have for every non-constant Lipschitz 1 function $f$

$$
\limsup _{N \rightarrow \infty}\left(2 \lambda_{N} \log \log \lambda_{N}\right)^{-1 / 2} \sum_{k=1}^{N} d_{k}\left(f\left(\frac{S_{k}}{\sqrt{k}}\right)-E f\left(\frac{S_{k}}{\sqrt{k}}\right)\right)=1 \quad \text { a.s. }
$$

where $\lambda_{N}$ is defined by (2.8). 
The basic new element in Theorems 2-4 is condition (2.7), which is weaker than the coefficient condition (2.4) in Theorem 1 and is very similar to Kolmogorov's classical condition for the LIL. Condition (2.7) permits a faster growing sequence $D_{N}$ (and thus it yields a stronger result) than the sequence $D_{N}=\exp \left((\log N)^{\alpha}\right), 0<\alpha<1$ in Proposition 1. In fact, (2.7) is satisfied if

$$
D_{N}=\exp \left(\log N /(\log \log N)^{\alpha}\right) \quad(\alpha>3) .
$$

As a matter of fact, condition (2.7) is essentially sharp: for $D_{N}$ as in (2.9) the conclusion of Theorem 4 fails with $0<\alpha<1$. Thus the 'dividing line' sequence in a.s. central limit theory is (2.9) and the change of behaviour happens in the interval $1<\alpha<3$. The critical value of $\alpha$ remains open.

\section{Proofs}

In what follows, we will only sketch the proofs of our results; for the detailed proof we refer to Hörmann (2005a) and (2005b). We use two different approaches to get Theorem 1 and Theorems 2-4.

\subsection{First approach}

To simplify the notation we set

$$
\xi_{k}=f\left(\frac{S_{k}}{a_{k}}-b_{k}\right)-E f\left(\frac{S_{k}}{a_{k}}-b_{k}\right) .
$$

Without loss of generality we may assume $|f| \leq 1$ and letting $X_{k}^{\prime}=X_{k}-\left(a_{k} b_{k}-\right.$ $\left.a_{k-1} b_{k-1}\right)(k=1,2, \ldots)$, we can also assume $b_{k}=0, k \geq 1$. From (2.1) it follows that the conclusion of Theorem 1 is equivalent to

$$
P\left\{\left|\sum_{k=1}^{N} d_{k} \xi_{k}\right|>\varepsilon D_{N} \quad \text { i.o. }\right\}=0 \quad \text { a.s. for all } \varepsilon>0 \text {. }
$$

The next lemma provides information on the covariances of the random variables $\xi_{k}$.

Lemma 2 Let $X_{1}, X_{2}, \ldots$ be independent random variables with partial sums $S_{n}$ and assume that for some numerical sequences $a_{n}>0$ and $b_{n}=0$ relations (2.2) and (2.3) are satisfied. Then there exist constants $c, \alpha>0$ such that for every bounded Lipschitz 1 function $f$ we have

$$
\left|E \xi_{k} \xi_{l}\right| \leq c(k / l)^{\alpha} \quad(1 \leq k \leq l) .
$$

Using the last lemma, the Markov inequality and (2.4) we can show for any $\eta<\rho$

$$
P\left\{\left|\sum_{k=1}^{N} d_{k} \xi_{k}\right|>\varepsilon D_{N}\right\} \ll \frac{1}{\varepsilon^{2}}\left(\log D_{N}\right)^{-\eta}
$$


(Here $a_{n} \ll b_{n}$ means $\lim \sup _{n}\left|a_{n} / b_{n}\right|<\infty$.) Choose a sequence $N_{1}, N_{2}, \ldots$ such that

$$
\sum_{i \geq 1}\left(\log D_{N_{i}}\right)^{-\eta}<\infty
$$

Then the Borel-Cantelli Lemma implies that (2.5) holds along the subsequence $N_{1}, N_{2}, \ldots$ In order to prove Theorem 1 it remains to estimate the oscillation of $\sum_{k=1}^{N} d_{k} \xi_{k}$ between the $N_{i}$ 's. It is clear that if the gaps between $N_{i}$ and $N_{i+1}$ are too large, the oscillation will get out of control. On the other hand, we are forced to choose a rapidly growing sequence $\left(N_{i}\right)$ in order to get (3.3). At this point the reason for assuming the condition $\alpha<1 / 2$ for the weights of Berkes and Csáki (1.8) becomes clear. Simple analysis shows that for these $d_{k}$ we have

$$
d_{k}=O\left(\frac{D_{k}}{k\left(\log D_{k}\right)^{(1-\alpha) / \alpha}}\right)
$$

If $\alpha<1 / 2$, then we can choose $\eta>1$ in (3.2)-(3.3) and thus for some $\delta>0$ we have $\eta(1-\delta)>1$. This assures that for a subexponential sequence $D_{N_{i}} \sim e^{i^{1-\delta}}$ the series in (3.3) converges. The fact that the sequence $\left(D_{N_{i}}\right)$ is subexponential provides that the oscillation of $\sum_{k=1}^{N} d_{k} \xi_{k}$ between the $N_{i}$ 's is sufficiently small. The crucial tool for an improvement of the ASCLT is contained in the following moment inequality, which is similar to the well known Rosenthal inequality. (Cf. Petrov, 1995: p. 59).

Lemma 3 Let $X_{1}, X_{2}, \ldots$ be independent random variables with partial sums $S_{n}$. Assume that (2.2) and (2.3) are satisfied for some sequences $a_{n}>0$ and $b_{n}=0$. Further let $f$ be a bounded Lipschitz, 1 function. Then for every $p \in \mathbb{N}$ we have

$$
E\left|\sum_{k=1}^{N} d_{k}\left(f\left(\frac{S_{k}}{a_{k}}\right)-E f\left(\frac{S_{k}}{a_{k}}\right)\right)\right|^{p} \leq C_{p}\left(\sum_{1 \leq k \leq l \leq N} d_{k} d_{l}\left(\frac{k}{l}\right)^{\alpha}\right)^{p / 2},
$$

where $C_{p}=C\left(p, X_{1}, \alpha\right)$ ( $\alpha$ is the constant in (3.1)) does not depend on $N$.

With Lemma 3 and the Markov inequality we can improve (3.2). We set $\eta=\rho / 2$ and get

$$
P\left\{\left|\sum_{k=1}^{N} d_{k} \xi_{k}\right|>\varepsilon D_{N}\right\} \leq \frac{c(m)}{\varepsilon^{2 m}}\left(\log D_{N}\right)^{-m \eta} \quad(m \geq 1) .
$$

Choosing $N_{j}$ such that $D_{N_{j}} \sim \exp (\sqrt{j})$ and $m>2 / \rho$ gives in conjunction with the Borel-Cantelli lemma

$$
\lim _{j \rightarrow \infty} \frac{1}{D_{N_{j}}} \sum_{k=1}^{N_{j}} d_{k} \xi_{k}=0 \text { a.s. }
$$

A simple calculation shows that if $N_{j} \leq N<N_{j+1}$

$$
\frac{1}{D_{N}}\left|\sum_{k=1}^{N} d_{k} \xi_{k}\right| \leq \frac{1}{D_{N_{j}}}\left|\sum_{k=1}^{N_{j}} d_{k} \xi_{k}\right|+\frac{D_{N_{j+1}}}{D_{N_{j}}}-1 \quad \text { a.s. }
$$

Since $D_{N_{j+1}} / D_{N_{j}} \rightarrow 1$ the convergence of the subsequence implies that the whole sequence converges a.s. 


\subsection{Second approach}

The approach to Theorems $2-4$ is based on a blocking technique. The proof and the corresponding lemmas are very technical and therefore we will just illustrate the main idea, which is simple.

Lemma 4 Let $X_{1}, X_{2}, \ldots$ be i.i.d. r.v.'s with $E X_{1}=0$ and $E X_{1}^{2}=1$ and let $S_{n}=$ $X_{1}+\cdots+X_{n}$. Further let $d_{k}=L(k) / k$, where $L$ is slowly varying at infinity and let $f$ be a bounded Lipschitz 1 function. Then

$$
\operatorname{Var}\left(\sum_{k=1}^{N} d_{k} f\left(\frac{S_{k}}{\sqrt{k}}\right)\right) \gg \sum_{k=1}^{N} k d_{k}^{2} .
$$

Lemma 4 shows that Theorem 2 is a consequence of Theorem 4, since (2.7) and (3.4) imply that

$$
D_{N} /\left(\lambda_{N} \log \log \lambda_{N}\right)^{1 / 2} \rightarrow \infty .
$$

The idea of the proof of Theorems 3 and 4 is this: We partition $\mathbb{N}$ into disjoint blocks: $\mathbb{N}=A_{1} \cup B_{1} \cup A_{2} \cup B_{2} \ldots$, where

$$
B_{j}=\left\{2^{p_{j}}+1, \ldots, 2^{q_{j}}\right\} \quad \text { and } \quad A_{j}=\left\{2^{p_{j}^{\prime}}+1, \ldots, 2^{q_{j}^{\prime}}\right\} .
$$

The exponents $p_{j}, q_{j}, p_{j}^{\prime}$ and $q_{j}^{\prime}$ are defined such that the number of elements in $B_{j}$ and in the $A_{j}$ is increasing very fast and that $\left|B_{j}\right|$ is much larger than $\left|A_{j}\right|$. Next we define the random variables

$$
Z_{j}:=\sum_{k \in B_{j}} d_{k} \xi_{k} \quad \text { and } \quad R_{j}:=\sum_{k \in A_{j}} d_{k} \xi_{k} \quad(j \geq 1) .
$$

Then clearly

$$
\sum_{j=1}^{n}\left(Z_{j}+R_{j}\right)=\sum_{k=1}^{2^{q_{n}}} d_{k}\left(f\left(\frac{S_{k}}{\sqrt{k}}\right)-E f\left(\frac{S_{k}}{\sqrt{k}}\right)\right) .
$$

The idea is to approximate the random process $\left\{Z_{j}, j \geq 1\right\}$ by an independent process $\left\{Z_{j}^{*}, j \geq 1\right\}$. We define

$$
Z_{j}^{*}=\sum_{k \in B_{j}} d_{k} \xi_{k}^{j} \quad(j \geq 1)
$$

with

$$
\xi_{k}^{j}=f\left(\frac{S_{k}-S_{l_{j}}}{\sqrt{k}}\right)-E f\left(\frac{S_{k}-S_{l_{j}}}{\sqrt{k}}\right) \quad \text { for } k \in B_{j},
$$

where $l_{j}$ is the largest integer contained in $B_{j-1}$. Then clearly by its definition the random variables $Z_{j}^{*}$ are independent. Note that the faster the number of elements in the blocks $A_{j}$ and $B_{j}$ grow the smaller will be the error which we make by replacing $\xi_{k}$ by $\xi_{k}^{j}$. However, too large blocks will cause a higher oscillation of the $Z_{j}$ 's and $R_{j}$ 's, which leads to a worse reminder term. Therefore an optimal choice of $\left|A_{j}\right|$ and $\left|B_{j}\right|$ is very important, in order to get optimal results. 
Now we derive in a first step a CLT and an LIL for the sequences $\left\{Z_{j}^{*}, j \geq 1\right\}$ via Lyapunov's theorem and Kolmogorov's classical LIL (cf. Petrov, 1995: Theorem 4.9 and Theorem 7.1).

In the second step we estimate the difference between $Z_{j}^{*}$ and $Z_{j}$. It turns out that it does not disturb the limiting behavior, i.e. the approximation is close enough. Further we show that the contribution of $\sum_{j=1}^{n} R_{j}$ to

$$
\sum_{k=1}^{2^{q_{n}}} d_{k}\left(f\left(\frac{S_{k}}{\sqrt{k}}\right)-E f\left(\frac{S_{k}}{\sqrt{k}}\right)\right)
$$

is negligible for our purposes. This proves the theorems along the subsequence $\left(2^{q_{n}}\right)_{n \geq 1}$. Finally it remains to show that the fluctuation of the partial sums between $2^{q_{n}}$ and $2^{q_{n+1}}$ is small enough.

\section{Acknowledgements}

The author would like to thank Prof. István Berkes for his great support and two anonymous referees for carefully reading the paper and improving the first version.

\section{References}

[1] Berkes, I. (1998): Results and problems related to the pointwise central limit theorem. In Asymptotic methods in probability and statistics (Ottawa, ON, 1997) (pp. 59-96). Amsterdam: North-Holland.

[2] Berkes, I. and Csáki, E. (2001): A universal result in almost sure central limit theory. Stochastic Processes and their Applications, 94, 105-134.

[3] Berkes, I. and Dehling, H. (1993): Some limit theorems in log density. The Annals of Probability, 21, 1640-1670.

[4] Brosamler, G. A. (1988): An almost everywhere central limit theorem. Math. Proc. Cambridge Philos. Soc., 104, 561-574.

[5] Chandrasekharan, K. and Minakshisundaram, S. (1952): Typical Means. Oxford University Press.

[6] Csörgó, M. and Révész, P. (1981): Strong Approximations in Probability and Statistics. Budapest: Academic Press.

[7] Erdôs, P. and Hunt, G. (1953): Changes of signs of sums of random variables. Pacific J. Math., 3, 673-687.

[8] Hörmann, S. (2005a): Summation methods and the almost sure central limit theorem. Preprint.

[9] Hörmann, S. (2005b): An extension of almost sure central limit theory. Accepted for publication in Statist. Probab. Lett.

[10] Lacey, M. T. and Philipp, W. (1990): A note on the almost sure central limit theorem. Statist. Probab. Lett., 9, 201-205.

[11] Lévy, P. (2003): Théorie de l'addition des variables alétoires (12 ed.). Jacques Gabay. 
[12] Peligrad, M. and Révész, P. (1991): On the almost sure central limit theorem. In Almost everywhere convergence, II (Evanston, IL, 1989) (pp. 209-225). Boston, MA: Academic Press.

[13] Petrov, V. (1995): Limit Theorems of Probability Theory. Oxford: Oxford Science Publications.

[14] Schatte, P. (1988): On strong versions of the central limit theorem. Math. Nachr., 137, 249-256.

[15] Weber, M. (2000): Un théorème central limite presque sûr à moments généralisés pour les rotations irrationnelles. Manuscripta Math., 101, 175-190. 